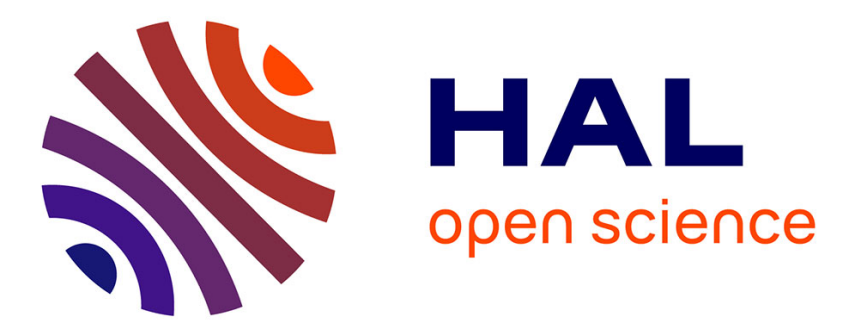

\title{
Vertical angular momentum transfer from accretion discs and the formation of large-scale collimated jets
}

F. Casse

\section{To cite this version:}

F. Casse. Vertical angular momentum transfer from accretion discs and the formation of largescale collimated jets. Plasma Physics and Controlled Fusion, 2008, 50, pp.4020. 10.1088/0741$3335 / 50 / 12 / 124020$. hal-00684064

\section{HAL Id: hal-00684064 \\ https://hal.science/hal-00684064}

Submitted on 30 Mar 2012

HAL is a multi-disciplinary open access archive for the deposit and dissemination of scientific research documents, whether they are published or not. The documents may come from teaching and research institutions in France or abroad, or from public or private research centers.
L'archive ouverte pluridisciplinaire HAL, est destinée au dépôt et à la diffusion de documents scientifiques de niveau recherche, publiés ou non, émanant des établissements d'enseignement et de recherche français ou étrangers, des laboratoires publics ou privés. 
35th EPS Conference on Plasma Physics

\title{
Vertical angular momentum transfer from accretion disks and the formation of large-scale collimated jets
}

\author{
F. Casse \\ AstroParticule \& Cosmologie (APC), Université Paris Diderot \\ UMR 7164 CNRS (Univ. Diderot, CEA, Obs. de Paris) \\ 10, rue A. Domon \& L. Duquet 75205 Paris Cedex 13, France \\ E-mail: fcasse@apc.univ-paris7.fr
}

\begin{abstract}
In this paper I present an overview of the favored scenario explaining the presence of twin cylindrical astrophysical jets in the vicinity of accretion disks. These jets are made of plasma and host large-scale magnetic fields. The twin jets flow away from the accreting system in opposite directions, perpendicular to the plane of the accretion disk. In the scenario presented in this paper, the accretion disk is interacting with the magnetic field in such a way that the disk angular momentum is removed from the disk and transported away along the magnetic field lines. Such a transport is the source of the jet phenomenon as the angular momentum is given back to a tiny amount of material extracted from the disk. This outflow is then powered by the disk rotation as the disk is able to enter an accretion motion where matter releases its gravitational energy. The angular momentum carried by the jet is actually present through the existence of an electric current. In the jet cylindrical geometry, the presence of this current is able to provide a collimating mechanism where the magnetic field pinches the plasma column. This mechanism is very close to the one acting in Tokamak reactors. Apart from explaining how the plasma outflow is able to be self-confined by the magnetic field present in the flow, this scenario is also able to explain how can jet mass be accelerated thanks to the MHD Poynting flux escaping from the disk. In this presentation I finally present the constraints arising from the scenario, in particular upon the turbulent transport coefficient required to get a steady structure.
\end{abstract}



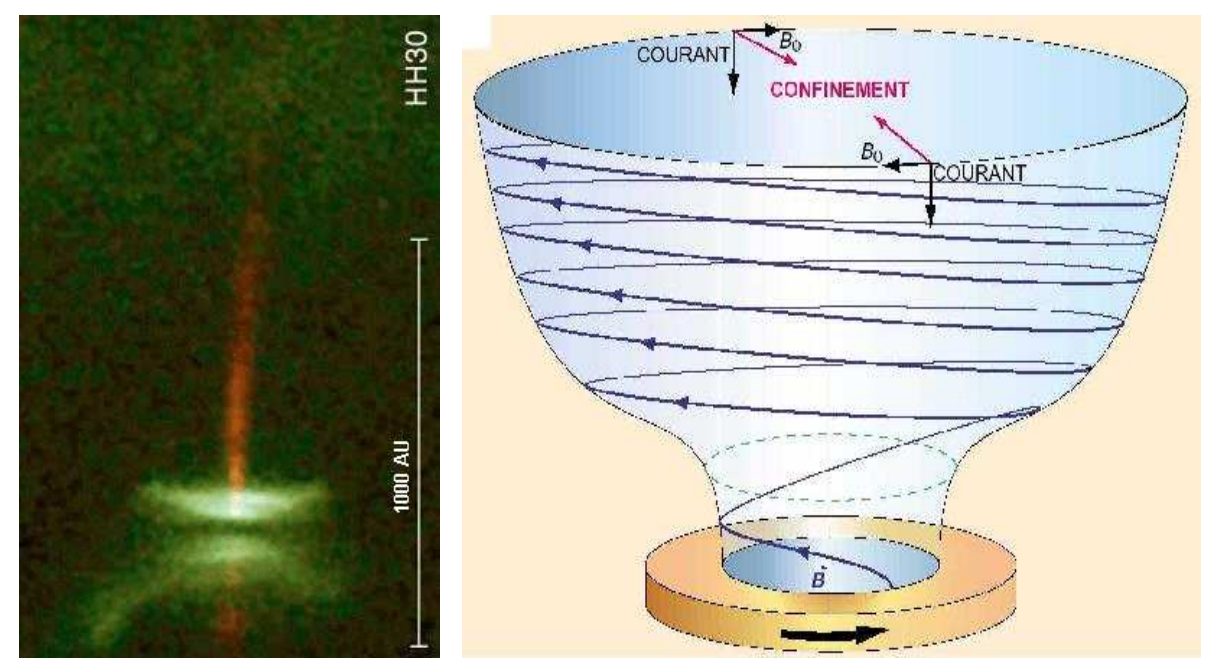

Figure 1. Left: Optical observation of a young forming star [4]. The protostar is surrounded by an accretion disk seen by the edge (black) and exhibit twin collimated, fast magnetized plasma jets. Right: Sketch of the accretion disk threaded by a twisted large-scale magnetic field leading to a vertical angular momentum transport from the disk to the jet (courtesy of J. Ferreira).

\section{A brief introduction to accretion disks dynamics}

Giant gas clouds are detected all over galaxies. Local fragmentation occurs within these clouds because of external perturbations such as supernovæ explosions or other gravitational perturbations. The fragmenting gas possess its own angular momentum with respect to a random axis. The self-gravity coming from the compressed gas is amplifying the gas compression. The angular momentum conservation forces the gas to rotate at faster rates up to a point where the centrifugal force can balance the gravity coming from the dense fragmenting core. This mechanism is at the origin of the birth of stars. The gravity being a spherically symmetrical force while the centrifugal force is axisymmetric, the only location where both forces may be in balance is the plane containing the dense core and being perpendicular to the rotation axis of the system. The resulting gas structure is thus a flatten differentially rotating disk. In order to balance gravity, the disk angular velocity has to be

$$
\Omega_{K}(r)=\sqrt{\frac{G M_{*}}{r^{3}}}
$$

which is called the Keplerian angular velocity $\left(G\right.$ is the gravitational constant, $M_{*}$ is the mass of the protostar and $r$ is the distance from the center of the protostar). Since we detect light coming from the disk itself, we do know that energy has to be released from the gas composing the disk. This energy can only come from the gravitational energy arising from the fall of disk material onto the protostar. In order to allow this gas motion called "accretion" (the overall disk material slowly falling toward the central object along spiraling streamlines), angular momentum has to be removed so that gravity can overcome the centrifugal barrier. The global accretion mass rate (amount of mass falling onto the protostar per second) is inferred from global spectroscopic observations and lead to values in low-mass protostars around 
$10^{-7}$ solar mass per year (see [5] for an observational review). Such accretion rates involve efficient dissipation mechanisms, likely to arise from turbulence. Indeed it has been considered that viscosity was the way to remove angular momentum thanks to anomalous viscous transport coefficient (ordinary coefficients would lead to very low accretion rate, at least ten orders of magnitude below what is observed). The resulting angular momentum transfer is occurring in the radial direction, thus unable to explain the presence of the observed large-scale jets flowing in the vertical direction. The wayout was to consider magnetic field presence within the disk, thus imposing to study the disk dynamics in the magnetohydrodynamics (MHD) framework (see [3, 19]).

The first jets have now been observed for more than ninety years. Considering the disk dynamical timescale, we can easily demonstrate that mass ejection from the disk and the associated angular momentum transfer has to occur continuously during many disk rotation periods. This has led disk theorists to assume in a first step that accretionejection phenomenon has to be explained in a steady framework. In addition, twin jets are often observed in these systems (jets having the same axis, same origin but flowing in opposite directions) so we can also assume that this kind of structure do possess a symmetric plane at the disk midplane (for a complete view see [10]).

In the following of this paper, I will mainly focus on analytical results arising from accretion-ejection structures. Numerous time-dependent MHD simulations have been performed in order to either confirm analytical predictions regarding disks and jets or to describe the behavior of such structures. The reader is invited to look at [20] for a review on numerical simulations.

\section{MHD Accretion mechanism}

\subsection{Accretion and turbulence}

The set of MHD equations used to describe the accretion-ejection structures are the mass and momentum conservation and induction equation that, in a steady, axisymmetric framework reads in cylindrical coordinates $(r, \varphi, z)$

$$
\begin{array}{ll}
\nabla \cdot \rho \mathbf{v} & =0 \\
\rho \mathbf{u} \cdot \nabla \mathbf{u} & =-\nabla P-\rho \nabla \Phi_{G}+\mathbf{J} \times \mathbf{B}+\nabla \cdot[T] \\
\nabla \times(\mathbf{v} \times \mathbf{B}) & =\nabla \times([\eta] \cdot \mathbf{J})
\end{array}
$$

where $\rho$ is the plasma density, $\mathbf{v}$ is the plasma velocity, $\mathbf{B}$ is the magnetic field, $\mu_{o} \mathbf{J}=\nabla \times \mathbf{B}$ is the current density, $P$ is the thermal pressure and $\Phi_{G}$ is the gravitational potential of the central object. The energy equation involves both local heating and cooling (radiative losses) that require both fluid approach and radiative transfer. A simplifying assumption is usually made as one considers the disk temperature to be constant over a given disk scale height (temperature thus only varies along the radius in the disk). The disk and jet plasma is considered as a perfect gas as $P=\rho C_{s}^{2}$. Let us note that we have included in these equations terms arising from turbulence such as anomalous viscous torque $\nabla \cdot[T]$ where $[T]$ is the viscous tensor and an anomalous magnetic resistivity tensor $[\eta]$. Indeed, in a steady framework, the radial component of the magnetic induction equation states that within the disk

$$
\eta_{p} \frac{\partial B_{r}}{\partial z}=-v_{r} B_{z}+v_{z} B_{r} \simeq-v_{r} B_{z}>0
$$

since in the disk the radial velocity is much larger than the vertical velocity $\left(\eta_{p}\right.$ is the resistivity component related to the poloidal field). The accretion motion $\left(v_{r}<0\right)$ 
thus imposes that resistivity has to occur within the disk and that this accretion will provoked a bending of the poloidal magnetic field lines $\left(B_{r}>0\right)$. MHD instabilities in accretion disks have been investigated for several decades in order to explain the presence of anomalous transport phenomena. The most famous one is the magnetorotational instability (MRI) that occurs in any magnetized disk (see the review by [2]). Indeed, it has been demonstrated since [1] that magnetized accretion disks are prone to turbulence because of the differential rotation law leading to a tearing of the local magnetic field. If a part of a given magnetic field line is anchored to an inner disk region rotating at a higher velocity than an outer part linked to the same field line, the magnetic field will then be torn, leading to a turbulence occurring inside the disk. Let us note that other instabilities have been discovered within accretion disk such as Rossby wave instability and accretion-ejection instability (see e.g. [17, 15, 22, 23]). When instabilities are at work within the disk, the dissipation is occurring on very short timescales so the accretion remains stationary while the plasma is prone to anomalous resistivity. So far there has been no physically motivated analytical expression of these anomalous transport coefficient. The jet arising from the disk is believed to be prone to ideal MHD since its mass density is much weaker than in the disk. The vertical profile of the anomalous transport coefficients is thus expected to decrease from its maximal value at the disk midplane to a zero value at the disk surface $(z=h)$.

\subsection{A turbulent magnetic torque}

The presence of resistivity in the disk has an important influence on the behavior of the disk magnetic field (see [14, 12] for the first Ohmic diffusivity and ambipolar diffusion dominated disk models). Indeed, if one writes the radial component of the induction equation at the disk midplane $(z=0)$, one easily gets

$$
B_{z}\left(\Omega-\Omega_{*}\right)=-\eta_{t}\left(\frac{\partial B_{\varphi}}{\partial z}\right)_{z=0}
$$

where $\eta_{t}$ is the resistivity component related to the toroidal field, $\Omega$ is the angular velocity of the plasma while $\Omega_{*}$ is the local angular velocity of the magnetic field lines. The disk rotation is driving the magnetic field line rotation but as turbulence is present in the disk, the disk matter do rotate at a larger angular velocity than magnetic field lines $\left(\Omega>\Omega_{*}\right)$ since the plasma can diffuse through the magnetic field lines in all directions. The velocity shift between matter and magnetic field has an effect on the magnetic field as it can be observed in Eq.(6) as the magnetic field becomes twisted by the disk rotation. The magnetic field feedback on disk material is the presence of a braking magnetic torque as

$$
(\mathbf{J} \times \mathbf{B}) \cdot \mathbf{e}_{\varphi} \simeq \frac{\partial B_{\varphi}}{\partial z} \frac{B_{z}}{\mu_{o}} \neq 0
$$

is negative in the disk. The magnetic field reaction to the faster rotating disk matter is then a toroidal braking trying to slow down the plasma. An alternative view of this interaction is the fact that disk angular momentum can be removed by the action of the magnetic torque and thus allowing accretion of mass. The disk toroidal equilibrium expressed at the disk midplane ( $\varphi$ component of Eq.3), provides an estimate of the accretion velocity $([6])$

$$
\frac{-v_{r}(z=0)}{C_{s}}=-\frac{h}{B_{z}} \frac{\partial B_{\varphi}}{\partial z} \frac{\Omega_{K}}{\beta_{P} \Omega_{o}}+\alpha_{v} \frac{h}{r} \simeq-\frac{B_{\varphi}^{+}}{\beta_{P} B_{z}}+\alpha_{v} \frac{h}{r}
$$


where $\beta_{P}$ is the usual ratio of the gas pressure to magnetic pressure at the disk midplane (the "+" index stands for quantities evaluated at the disk surface $z=h$ while subscript "o" stands for estimates done at the disk midplane). The two terms contributing to the accretion motion are the magnetic torque and the viscous torque. The magnetic torque is often much larger than the viscous torque as observed disk are thin $(h \ll r)$ provided that the $\beta_{P}$ parameter is not larger than $r / h$. It appears then that magnetized accretion disk angular momentum transport will occur thanks to the magnetic field, thus along the magnetic field lines as angular momentum conservation provides

$$
\rho \mathbf{v}_{p} \cdot \nabla\left(\Omega r^{2}\right)=\mathbf{B}_{p} \cdot \nabla\left(\frac{r B_{\varphi}}{\mu_{o}}\right)+(\nabla \cdot \mathbf{T}) \cdot \mathbf{e}_{\varphi}
$$

The removed disk angular momentum is converted by the magnetic field into toroidal magnetic field.

\subsection{MHD accretion disk equilibrium}

We have seen in the previous paragraphs that the dynamic of the disk is very likely to produce both bended $\left(B_{r}^{+}>0\right)$ and twisted $\left(B_{\varphi}^{+}<0\right)$ magnetic field. The presence of such component has an impact on the vertical disk equilibrium (see e.g. [24, 9]). Indeed the vertical momentum conservation coming from Eq.(3) leads to a quasi magneto-hydrostatic equilibrium (we have $v_{z} \ll C_{s}$ because of the thin disk geometry) if the vertical thermal pressure gradient is able to balance both the gravitational and magnetic pinching of the disk. Integrating this equilibrium over the disk scale height leads to the relation

$$
P_{o} \simeq \frac{B_{r}^{+2}+B_{\varphi}^{+2}}{2 \mu_{o}}+\int_{0}^{h} \rho \Omega_{K}^{2} z d z+\left[\rho \frac{u_{z}^{2}}{2}\right]_{0}^{h}-\int_{0}^{h} u_{z}^{2} \frac{\partial \rho}{\partial z} d z
$$

Since the last two terms are negative, it is obvious that the condition for the disk to remain in balance is simply that

$$
\beta_{P} \geq \frac{B_{r}^{+2}+B_{\varphi}^{+2}}{B_{o}^{2}}
$$

If this condition was not to be fulfilled then the accretion disk would be compressed, reducing its height and thus increasing the gas pressure so that it can again verify the previous condition.

\section{The connection between magnetized disk and MHD jets}

\subsection{Magneto-centrifugal acceleration}

We have seen in the previous section that the presence of the magnetic field plays an important role in the angular momentum removal from the disk. This torque is also the key to drive an outflow from the surface of the disk. Indeed, if one projects the magnetic force along the total magnetic field, one easily gets

$$
(\mathbf{J} \times \mathbf{B}) \cdot \mathbf{B}_{p}=-(\mathbf{J} \times \mathbf{B}) \cdot \mathbf{B}_{\varphi}
$$

which means that the sign of the magnetic torque is always the same than the sign of the projection of the magnetic force along the poloidal magnetic field lines $\left(B_{\varphi}<0\right)$. So as long as the magnetic torque is braking the disk material, the poloidal magnetic 
field will pinch the accretion disk, thus preventing any mass outflow from the disk. The key to get a transition from an accretion motion to an ejection phenomena is simply to have a magnetic torque changing its sign near the disk surface. Indeed, once the magnetic torque starts to accelerate mass above the disk (in the jet region), the poloidal magnetic force will also start to speed up mass along the magnetic surfaces. Moreover, the toroidal acceleration also increases the specific angular momentum of mass thus increasing the centrifugal force. The outcome of this situation is that two poloidal forces do accelerate matter in the jet: the poloidal Lorentz force and the centrifugal force, both of them being directly related to a positive magnetic torque. This kind of mechanism is called the magneto-centrifugal acceleration; it is the corner stone of the jet acceleration. Nevertheless such magnetic topology enabling the magnetic torque to switch its sign from negative in the disk (braking) to positive in the jet (acceleration) has some important impact on the amplitude of the MHD turbulence occuring in the accretion disk.

It has been shown since [3] that magneto-centrifugal launching of a jet where the thermal pressure is believed not to play a significant role, is working only if $B_{r}^{+} / B_{o}>$ $\sqrt{3} / 3$. This minimal poloidal magnetic field line bending injected in Eq.(11) provides that $\beta_{P}$ has to be at least of order unity in order to provide a suitable disk equilibrium. Looking back at the vertical disk balance, we can see that gravity force is at least of the order of the magnetic pinching thus we can have an estimate of the disk sound speed by neglecting magnetic terms, namely $C_{s} \sim \Omega_{K} h$.

\subsection{Switching magnetic torque}

The magnetic torque in the disk is mainly driven by the radial density current in a thin accretion disk where magnetic quantities derivatives along $z$ are much larger than along the radial direction $\left(J_{z} \ll J_{r}\right)$. Indeed, writing down the magnetic torque leads to

$$
(\mathbf{J} \times \mathbf{B}) \cdot \mathbf{e}_{\varphi}=J_{z} B_{r}-J_{r} B_{z} \simeq-J_{r} B_{z}
$$

where $J_{r}$ is positive at the disk midplane. Thus a reversal of the magnetic torque near the disk surface imposes that the radial density current tends toward zero near the disk surface [7]. Writing down the resistive induction equation controlling the behavior of $J_{r}$ in a thin disk gives [7]

$$
\eta_{t} J_{r}(z=h)=\eta_{t, o} J_{r, o}+r \int_{0}^{h} \mathbf{B}_{p} \cdot \nabla \Omega d z=\eta_{t, o} J_{r, o}(1-\Gamma)
$$

where $\eta_{t}$ is the resistivity tensor component related to the poloidal current (that may be different from the one dealing with the toroidal current, namely $\eta_{p}$ ). Imposing that the radial current tends toward zero at the disk surface implies that $\Gamma \simeq 1$. The radial rotation profile follows the Keplerian law, namely $\Omega \propto r^{-3 / 2}$. If one assumes that the angular mass velocity is constant over one disk scale height, we then end up with a constraint upon the relative amplitude of the toroidal to the radial magnetic field components at the disk surface, namely [8]

$$
-\frac{B_{\varphi}^{+}}{B_{r}^{+}} \simeq \frac{3 \mu_{o} \Omega_{o} h^{2}}{4 \eta_{t, o}}
$$

If this relation is verified in the disk, there will be a sign switch of the magnetic torque occurring near the disk surface and thus leading to a coexisting accretion flow (where the torque is negative) and ejection flow (positive torque). 


\subsection{Jet magnetic surface rotation}

Once a fraction of the accretion flow has reached the jet launching region, the flow enters an ideal MHD regime. In this regime the magnetic surface angular velocity becomes an invariant along the magnetic field surfaces. This velocity is related to disk surface quantities, namely

$$
\Omega_{*}=\Omega-\frac{v_{z} B_{\varphi}}{r B_{z}}
$$

The magneto-centrifugal acceleration is a quite efficient mechanism so that the jet flow escapes from the disk surface with velocities that are of the order of the disk sound speed (see the discussion in [8]). Replacing this estimate in the previous expression leads to an estimate of the constant angular velocity of magnetic surface whose surface cross the disk surface at radius $r$,

$$
\Omega_{*} \simeq \Omega_{o}-\frac{\Omega_{K} h B_{\varphi}^{+}}{r B_{o}}
$$

The magnetic surface whose constant angular velocity is $\Omega_{*}$ is anchored at the disk midplane at a radius $r_{o}$ which is smaller than $r$ because of the bending of the poloidal magnetic surfaces. The causality of the system is such that the angular velocity of the disk material at the footpoint $r_{o}$ of the magnetic surface has to be larger or equal than the magnetic surface angular velocity. Using Taylor expansions, it can be shown that $r_{o} / r \simeq B_{r}^{+} h / 2 r$, thus according to the disk angular velocity following a Keplerian profile, we have $\Omega_{o}\left(r_{o}\right) \simeq \Omega_{o}(r)\left(1+3 B_{r}^{+} h / 4 r\right)$. Equating $\Omega_{*}$ and $\Omega_{o}\left(r_{o}\right)$ provides an interesting causality constraint related to Eq.(15), namely ([8])

$$
-\frac{B_{\varphi}^{+}}{B_{r}^{+}} \sim 1
$$

Since the minimal bending criterion for jet launching states that $B_{r}^{+} / B_{o} \sim 1$, it is easy to see that the natural magnetic topology verifying all aforementioned constraints is then a disk where at its surface we have $-B_{\varphi}^{+} \sim B_{r}^{+} \sim B_{o}$. Such a magnetic topology is in agreement with a mild magnetic disk compression (enabling the thermal pressure to balance gravity and the magnetic action), with the minimal magnetic field line bending enabling the steady launching of the jet and with jet magnetic surfaces rotating at a smaller angular velocity compared to the disk angular velocity driving their rotation. Looking back at previous constraints, we can derive others interesting relations such as in Eq.(15) where the previous topology leads to

$$
\eta_{t, o} \sim \mu_{o} \Omega_{o} h^{2} \simeq \mu_{o} \Omega_{K} h^{2}
$$

The maximal disk magnetization is thus, looking at Eq.(11) $\beta_{P} \sim 2$, a disk where magnetic pressure is close to equipartition with the thermal pressure.

\section{Jet dynamics}

The jet dynamics is mainly controlled by the behavior of the current $I=r B_{\varphi} / \mu_{o}<0$. Indeed, we can easily rewrite the Lorentz force parallel to the poloidal magnetic field as a function of $I$,

$$
(\mathbf{J} \times \mathbf{B}) \cdot \mathbf{B}_{p}=-(\mathbf{J} \times \mathbf{B}) \cdot \mathbf{B}_{\varphi}=-\frac{B_{\varphi} B_{p}}{2 \pi r} \nabla_{/ /} I
$$

where $\nabla_{/ /}$is the derivative along a magnetic surface. We have already seen in the previous section that magneto-centrifugal acceleration is at work as soon as the 
magnetic torque becomes positive $\left(\nabla_{/ /} I>0\right)$. The current carried by the magnetic surface is thus used by the flow to provide a source of energy for the mass acceleration. Indeed, projecting the momentum conservation onto the magnetic surface leads to the specific Bernoulli invariant $E$ which provides a measure of the total amount of energy present on a magnetic surface, namely $([3,18,25,13,7])$

$$
E=\frac{v^{2}}{2}+\Phi_{G}+C_{s}^{2}-\Omega_{*} \frac{\mu_{o} I}{\sigma}
$$

where $\sigma$ is the mass to magnetic flux MHD invariant appearing in the MHD frozen-in law. The second and third terms are negligible once the flow is far from the disk so we easily see that if $I$ starts to increase, tending toward zero, we get an increase of the kinetic energy of the flow. The exact value of the Bernoulli invariant is determined by the various physical quantity values at the disk surface where the flow enters an ideal MHD regime. A rough estimate of this invariant at the disk surface is

$$
E \simeq-\frac{\Omega_{K}^{2} r^{2}}{2}+\frac{\Omega_{K} r B_{o}}{\sigma}=-\frac{\Omega_{K}^{2} r^{2}}{2}+\frac{\Omega_{K}^{2} r h}{\sqrt{\beta_{P}}} \frac{\rho_{o}}{\rho_{A}}
$$

where $\rho_{A}$ is the jet mass density at the Alfvén surface. The density once the flow reach the local Alfvén velocity has to be very small compared to the disk density (to insure $E>0$ ) so a condition to get a fast jet is to get a small amount of mass escaping from the disk. The magnetic field is twisted by the disk rotation, generating a MHD Poynting flux carrying the disk angular momentum along the magnetic field lines. This angular momentum is then given back to the flow in the jet region, enabling a strong acceleration because of the small amount of mass present in the jet.

The jet collimation is obtained in the same way than in Tokamaks reactors, namely by the action of the magnetic field $([16,11,21])$. Here astrophysical jets exhibit cylindrical geometry so that if one writes the expression of the radial magnetic force in cylindrical coordinates, one gets

$$
(\mathbf{J} \times \mathbf{B}) \cdot \mathbf{e}_{r}=-\frac{1}{\mu_{o}} \frac{\partial B_{z}^{2}}{\partial r}-\frac{\mu_{o}}{r} \frac{\partial}{\partial r} \frac{I^{2}}{r}+\frac{B_{z}}{\mu_{o}} \frac{\partial B_{r}}{\partial z}
$$

The radial current distribution is the key factor determining if the flow can recollimate and reach a cylindrical shape. Indeed, the jet widening above the disk has a tendency to dilute the poloidal magnetic field because of the $\nabla \cdot \mathbf{B}=0$ constraint. Thus the dominant role in the collimation is dedicated to the toroidal field responsible for the current carried by the jet. If the current distribution is suitable for collimation (namely a sufficiently increasing current) the "hoop stress" effect generated by the toroidal magnetic field will pinch the plasma jet, leading to the cylinder shape observed in astrophysical jets.

\section{Concluding remarks}

Over the last three decades, important progresses have been made about the understanding of the astrophysical jet formation. In particular, the most successful model able to reproduce some observations regarding such systems ([5]) displays now a clear scenario involving a large scale magnetic field interacting with an underlying accretion disk. The twisting of the magnetic field is responsible for the disk angular removal and transfer into the jet where a small amount of the disk material is ejected from the disk and accelerated thanks to current created by the twisting of the magnetic field. Despite these successes, some important issues remain as for instance the 
origin of the mandatory MHD disk turbulence. The related turbulent resistivity has to reach a high amplitude because of the constraints imposed to get a stationary outflow. Identifying the MHD instability able to trigger turbulence even with magnetic field near equipartition with thermal pressure and measuring the related turbulent transport coefficients will be quite a challenge in the forthcoming years.

\section{References}

[1] Balbus, S. A. \& Hawley, J.F. 1991, ApJ, 376, 223

[2] Balbus, S. A. 2003, Annual Review of Astronomy and Astrophysics, 41, 555

[3] Blandford, R. D. \& Payne, D. G. 1982, MNRAS, 199, 883

[4] Burrows, C.J. et al. 1996, ApJ, 473, 437

[5] Cabrit, S. 2007, Proceedings of the International Astronomical Union, Vol. 243, 203

[6] Casse, F. \& Ferreira, J. 2000, Astron. \& Astrop., 353, 1115

[7] Ferreira, J. 1997, Astron. \& Astrop., 319, 340

[8] Ferreira, J. \& Casse, F. 2008, submitted to MNRAS

[9] Ferreira, J. \& Pelletier, G. 1993, Astron. \& Astrop., 276, 637

[10] Frank, J., King A.R. \& Raine, D.J. 1985, Accretion power in astrophysics, Cambridge University Press

[11] Heyvaerts, J. \& Norman, C. 1989, ApJ, 347, 1055

[12] Königl, A. 1989, ApJ, 342, 208

[13] Li, Z.Y. 1995, ApJ, 444, 848

[14] Lovelace, R.V.E., Wang, J.C.L. \& Sulkanen, M.E. 1987, ApJ, 315, 504

[15] Lovelace, R.V.E., Li, H., Colgate, S. A., \& Nelson, A. F. 1999, ApJ, 513, 805

[16] Okamoto, I. 1975, MNRAS, 173, 373

[17] Papaloizou, J.C.B. \& Pringle, J.E. 1984, MNRAS, 208, 721

[18] Pelletier, G. \& Pudritz, R.E. 1992, ApJ, 394, 117

[19] Pudritz, R.E. 1985, ApJ, 293, 216

[20] Pudritz, R.E., Ouyed, R., Fendt, C. \& Brandendurg, A. 2007, Protostars and Planets V, University of Arizona Press, 277

[21] Pudritz, R.E., Rogers, C.S. \& Ouyed, R. 2006, MNRAS, 365, 1131

[22] Tagger, M. \& Pellat, R. 1999, Astron. \& Astrop., 349, 1003

[23] Tagger, M. \& Varniére, P. 2006, ApJ, 652, 1457

[24] Wang, J. C. L.; Sulkanen, M. E.; Lovelace, R. V. E. 1990, ApJ, 355, 38

[25] Wardle, M. \& Königl, A. 1993, ApJ, 410, 218 